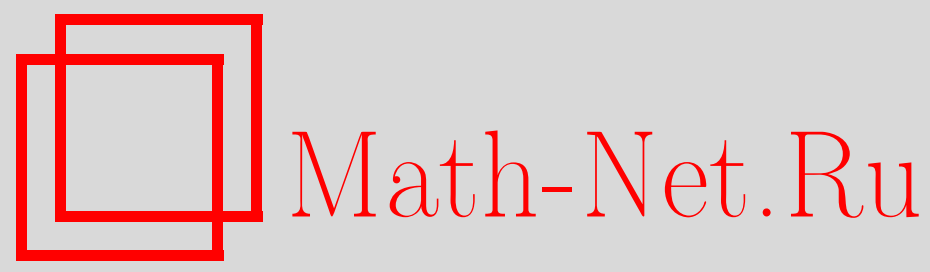

В. П. Белавкин, О квантовом стохастическом дифференциальном уравнении как краевой задаче Дирака, Матем. заметки, 2001, том 69, выпуск 6, 803-819

DOI: https://doi.org/10.4213/mzm695

Использование Общероссийского математического портала Math-Net.Ru подразумевает, что вы прочитали и согласны с пользовательским соглашением http://www . mathnet.ru/rus/agreement

Параметры загрузки:

IP : 54.157 .27 .8

26 апреля 2023 г., 14:55:03

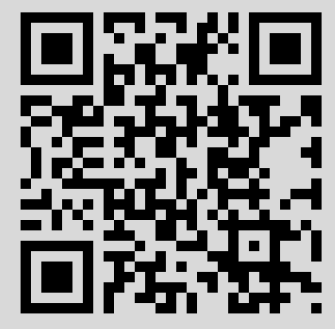




\title{
О КВАНТОВОМ СТОХАСТИЧЕСКОМ ДИФФЕРЕНЦИАЛЬНОМ УРАВНЕНИИ КАК КРАЕВОЙ ЗАДАЧЕ ДИРАКА
}

\section{В.П. Белавкин}

\begin{abstract}
В статье доказано, что квантовая стохастическая эволюция, порождаемая процессом числа частиц, унитарно эквивалентна краевой задаче Дирака на полупрямой в расшшренном пространстве. Показано, что эта точно решаемая задача может быть получена из краевой задачи Шрёдингера для положительного реалистического гамильтониана в ультрарелятивистском пределе, соответствующем потоку частиц Дирака с асимптотически бесконечным импульсом. Эта задача стохастической аппроксимации сводится к квантовомеханической краевой задаче в расширенном пространстве. В статье также обсуждается вопрос о микроскопической обратимости времени.
\end{abstract}

Библиография: 7 названий.

1. Введение. Модели стохастической эволюции в гильбертовом пространстве недавно нашли интересные приложения в квантовой теории измерений [1]-[3]. В этой статье мы приведем пример того, как классический скачкообразный процесс может быть выведен из непрерьвной детерминированной квантовой консервативной динамики с начальньм условием в виде чистого состояния. Как недавно было замечено в [2], кусочно-непрерывная унитарная стохастическая эволюция, управляемая квантовым пуассоновским процессом, эквивалентна уравнению Шрёдингера с зависящим от времени сингулярным гамильтонианом, а непрерьвная стохастическая унитарная эволюция, управляемая квантовым винеровским процессом, может быть получена как решение этой задачи в пределе типа центральной предельной теоремы. Здесь мы начнем с несингулярной стационарной динамики.

Сушествует обширная литература о стохастическом пределе, в которой квантовая стохастика выводится из несингулярных взаимодействий, описываемых задачей Коши для уравнения Шрёдингера, путем изменения масштабов пространственных переменных и времени. Вместо замены масштаба для потенциала взаимодействия мы рассматриваем сингулярное взаимодействие как краевое условие и получаем стохастическую динамику как ультрарелятивистский предел для соответствующей краевой задачи для уравнения Шрёдингера в гильбертовом пространстве бесконечного числа частищ. Мы докажем, что разрывная и непрерывная квантовая эволюции могут быть получены из физически мотивированной непрерьвной в сильном смысле унитарной эволюции путем решения краевой задачи в расширенном гильбертовом пространстве с чистым начальным состоянием.

Работа выполнена при поддержке гранта EPSRC GR/M66196. 
Сначала мы опишем краевую задачу, соответствующую стохастической эволюции с единственной сингулярностью, и найдем ультрарелятивистский предел. В этом случае кусочно-непрерывная и непрерывная стохастическая эволюции могут быть получены так же, как и в [2], [4]. Но перед тем как осуществить эту программу, мы рассмотрим простейшую унитарную модель, дающую "нефизические" решения задачи с гамильтонианом свободной частицы $h(p)=-p$. Эта простейшая модель в рамках метода вторичного квантования была использована для описания процесса непрерьвного квантового измерения [5]. Недавно Чеботарев [6] показал, что вторично квантованная гамильтонова модель в фоковском пространстве с условиями разрыва эквивалентна модели квантовой стохастической эволюции Хадсона-Партасарати [7] в случае коммутирующих операторозначных коэффициентов ${ }^{1}$. Наш подход свободен от предположения о коммутативности операторозначных коэффициентов уравнения Хадсона-Партасарати, и мы изучаем обратимый гамильтониан Дирака. Более того, мы доказьваем, что стохастическая модель может быть получена из положительного релятивистского гамильтониана как индуктивньй ультрарелятивистский предел на объединении гильбертовых пространств функций из класса Харди. Мы назьваем этот предел индуктивной стохастической аппроксимачией.

2. Простейшая гамильтонова модель. Здесь на примере простейшей модели мы покажем, что задача с зависящим от времени стохастическим гамильтонианом может быть интерпретирована как представление взаимодействия самосопряженной краевой задачи для уравнения Шрёдингера, описьвающего сильно непрерывную унитарную группу.

Пусть $\mathscr{H}$ - гильбертово пространство, $H$ - ограниченньй снизу самосопряженный оператор и $S$ - унитарный оператор в $\mathscr{H}$, не обязательно коммутирующий с $H$. Оператор $H$, называемый гамильтонианом, является генератором консервативной эволюции квантовой системы, описьваемой уравнением Шрёдингера $i \hbar \partial_{t} \eta=H \eta$, a оператор $S$, назьваемьй оператором рассеяния, описывает унитарные квантовые скачки $\eta \mapsto S \eta$ векторов состояний $\eta \in \mathscr{H}$, вызванных сингулярным потенциалом взаимодействия в системе, которая при отсутствии скачков совершает непрерьвную унитарную эволюцию

$$
\eta \mapsto e^{-\frac{i}{\hbar} t H} \eta
$$

Как пример такого скачка мы рассмотрим сингулярньй гамильтониан модели фон Неймана для непрерывного косвенного измерения координаты квантовой частицы $x \in \mathbb{R}$, регистрируемой прибором в точке $y \in \mathbb{R}$. Эта модель может быть описана [2], [4] с помощью оператора сдвига $S$ точки $y$ на $x$ как умножение на $\hat{\sigma}$, где $\sigma(x)=e^{x \partial_{y}}-$ скачок в гильбертовом пространстве $L^{2}\left(\mathbb{R}^{2}\right)$ абсолютно интегрируемых с квадратом функций $\eta(x, y)$, не коммутирующий с гамильтонианом свободной эволюции

$$
H=\frac{1}{2}\left(y^{2}-\partial_{x}^{2}\right)
$$

\footnotetext{
${ }^{1}$ В работе [6] для уравнения Хадсона-Партасарати с коммутирующими коэффициентами построено явное решение, для которого найдено нетривиальное краевое условие в фоковском пространстве, описывающее операторозначные скачки амплитуды и фазы. Там же доказана симметричность соответствующей краевой задачи с произвольными некоммутирующими коэффициентами. Отметим, что до работы [6] интерпретация уравнений, рассматриваемых в квантовой теории вероятностей, как симметричных краевых задач в фоковском пространстве была неизвестна. Прим. переводчика.
} 
Такой гамильтониан используется, например, для описания системы “квантовых частищ плюс измерительньй аппарат" .

Обычно предполагается, что квантовые скачки происходят в случайные моменты времени $t=s$ с заданной плотностью вероятности $\rho(s)>0$ на положительной полупрямой $\mathbb{R}^{+}, \int_{0}^{\infty} \rho(s) d s=1$. Если $H$ и $S$ коммутируют, скачкообразный процесс стохастической эволюции по $t$ может быть формально описан уравнением Шрёдингера, зависящим от времени $i \hbar \partial_{t} \chi(t)=H_{s}(t) \chi(t)$ с сингулярным стохастическим гамильтонианом

$$
H_{s}(t)=H+i \hbar \delta_{s}(t) \ln S
$$

где $\delta_{s}(t)=\delta(t-s)=\delta_{t}(s)-\delta$-функция Дирака переменной $z=s-t$. Действительно, интегрируя гамильтониан $H_{r}(s)$ по $r$ от 0 до $t$ при фиксированном $s \in \mathbb{R}$, нетрудно получить

$$
V(t, s)=e^{-\frac{i}{\hbar}} \int_{0}^{t} H_{s}(r) d r=e^{-\frac{i}{\hbar} t H} S^{\Delta_{0}^{t}(s)}=e^{\frac{i}{\hbar}(s-t) H} S^{\Delta_{0}^{t}(s)} e^{-\frac{i}{\hbar} s H},
$$

где $\Delta_{0}^{t}(s)=\int_{0}^{t} \delta_{r}(s) d r$ отождествляется с индикаторной функцией $1_{[0, t)}$ интервала $[0, t)$ для $t>0$ (при $t \leqslant 0$ скачок равен нулю, если $s>0$ ). Правая часть является оператором унитарной стохастической эволюции $V(t, s)$, что остается справедливым и в случае, если операторы $H$ и $S$ не коммутируют. Начальная эволющия $V(t, s)=e^{-\frac{i}{\hbar} t H}$ консервативна и непрерьвна при $t \in[0, s)$, затем происходит квантовьй скачок $S$ при $t=s$, и при $t>s$ эволюция, описьваемая гамильтонианом $H$, вновь непрерьвна. Как было замечено в [2], строгая форма стохастического уравнения Шрёдингера, которая дает такие решения для некоммутирующих $H$ и $S$ в положительном направлении времени $t$, является линейньп стохастическим уравнением Ито

$$
d_{t} V(t, s)+\frac{i}{\hbar} H V(t, s) d t=(S-I) V(t, s) d 1_{t}(s), \quad t>0, \quad V(0, s)=I .
$$

Здесь $d_{t} V(t, s)=V(t+d t, s)-V(t, s)$ является упреждаюшим дифференциалом, соответствующим инфинитезимальному приращению $d t>0, d 1_{t}(s)=\Delta_{0}^{d t}(s-t)$ - это индикаторная функция $\Delta_{t}^{d t}(s)=1_{[t, t+d t)}(s)$, а $t \mapsto 1_{t}(s)=1_{0}(s-t)$ - упреждающее приращение функции Хэвисайда, где $1_{0}=1_{(-\infty, 0)}$. Уравнение $(2.2)$ означает, что $t \mapsto V(t)$ при фиксированном $s=z$ удовлетворяет обычному уравнению Шрёдингера $i \hbar \partial_{t} V(t)=H V(t)$, если $t \neq s$ при $d 1_{t}(s)=0$ для достаточно малых $d t(d t<s-t$, если $t<s$, и любого $d t>0$, если $t>s$ ), в тоже время при $t=s$ происходит скачок $d_{t} V=\left.(S-I) V d 1_{t}(s)\right|_{t=s}=1 \gg d t$. Интегрируя $d_{z} \chi(z)=d_{z} V(z) \eta$ в области определения оператора $H$ сначала от 0 до $z=s$ с начальным условием $\chi(0)=\eta$, а затем от $s$ до $t$ с начальньм условием $\chi\left(s_{+}\right):=\lim _{z \searrow s} \chi(z)=S \chi(s)$, нетрудно получить решение в виде $\chi(t, s)=V(t, s) \eta$, где

$$
V(t, z)=e^{-\frac{i}{\hbar} t H} S(z)^{1_{[0, t)}(z)}, \quad S(z)=e^{\frac{i}{\hbar} z H} S e^{-\frac{i}{\hbar} z H}
$$

без предположения коммутативности $H$ и $S$.

Теперь мы докажем, что скачкообразная стохастическая эволюция $V(t)$ может интерпретироваться в представлении взаимодействия $V(t) \chi^{0}=e^{\frac{i}{\hbar} t h(\hat{p})} \chi^{t}$ для детерминированной сильно непрерьвной шрёдингеровской эволюции $\chi^{0} \mapsto \chi^{t}$ для функций пере-

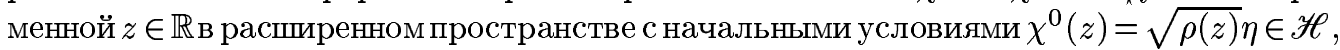
локализованных при $z>0: \chi^{0}(z)=0$ при $z \leqslant 0$. Здесь $h(\hat{p})=-\hat{p}-$ гамильтониан свободной частицы, где $\hat{p}=-i \hbar \partial_{z}-$ момент, соответствующий переменной $z \in \mathbb{R}$ в расширенном пространстве. 
УТВЕРЖДЕНИЕ 2.1. Задача Коши для стохастического уравнения Шрёдингера с гамильтонианом (2.2) унитарно әквивалентна самосопряэсенной краевой задаче для уравнения Шрёдингера

$$
i \hbar \partial_{t} \chi^{t}(z)=\left(H+i \hbar \partial_{z}\right) \chi^{t}(z), \quad \chi^{t}\left(0_{-}\right)=S \chi^{t}(0)
$$

в гильбертовом пространстве $\mathscr{H} \otimes L^{2}(\mathbb{R})$ в том смысле, что стохастическая эволюиия $V(t)$ при $t>0$ совпадает с унитарным коииклом $V(t, z) \chi^{0}(z)=\chi^{t}(z-t)$, разрешающим краевую задачу (2.4) относительно пропагатора $e^{t \partial_{z}}$, действующего по переменной $z: \chi^{t}=e^{t \partial_{z}} V(t) \chi^{0} \quad \forall \chi^{0} \in \mathscr{H} \otimes L^{2}(\mathbb{R})$.

ДокАЗАТЕльство. Краевая задача (2.4) корректно поставлена на множестве гладких интегрируемых с квадратом $\mathscr{H}$-значных функций $\chi$ и симметрична, поскольку оператор $H$ является самосопряженным в силу краевого условия

$$
0=\left(\|\chi(-0)\|^{2}-\|\chi(0)\|^{2}\right)=2 \int \operatorname{Re}\left\langle\chi(z) \mid \chi^{\prime}(z)\right\rangle d z=\frac{2}{\hbar} \operatorname{Im}\langle\chi \mid \hat{h} \chi\rangle
$$

Более того, эта задача является самосопряженной, поскольку ее решение, очевидно, является унитарным, т.е.

$$
\chi^{t}(z)=e^{\frac{i}{\hbar} z H} \chi_{t}(z+t), \quad \chi_{t}(s)=S^{\Delta_{0}^{t}(s)} \chi_{0}(s),
$$

где $\chi_{0}(s)=e^{-\frac{i}{\hbar} H z} \chi^{0}(s)$. Действительно, подставляя $\chi^{t}(z)=e^{\frac{i}{\hbar} z H} \chi_{0}^{t}(z)$ в уравнение (2.4), мы получаем уравнение переноса $\partial_{t} \chi_{0}^{t}(z)=\partial_{z} \chi_{0}^{t}(z)$ с тем же краевьп условием $\chi_{0}^{t}(-0)=S \chi_{0}^{t}(0)$ и начальным условием $\chi_{0}^{0}=\chi_{0}$, соответствующим $\chi^{0} \in \mathscr{H} \otimes L^{2}(\mathbb{R})$. Эта простая краевая задача имеет очевидное решение $\chi_{0}^{t}(z)=\chi_{t}(z+t)$, где $\chi_{t}$ задано в (2.5) следующим образом:

$$
\chi_{t}(s)=S^{1_{(0, t]}(t-s)} \chi^{0}(s), \quad t>0, \quad \chi_{t}(s)=S^{-1_{[-t, 0)}(s)} \chi^{0}(s), \quad t<0 .
$$

Унитарность оператора $S^{\Delta_{0}^{t}(s)}$ в $\mathscr{H}$ и сдвига $e^{t \partial_{z}}$ в $L^{2}(\mathbb{R})$ влечет унитарность разрешающего отображения $V^{t}: \chi^{0} \mapsto \chi^{t}$ в $\mathscr{H} \otimes L^{2}(\mathbb{R})$,

$$
\left\|\chi^{t}\right\|^{2}=\left\|\chi_{t}\right\|^{2}=\left\|\chi_{0}\right\|^{2}=\left\|\chi^{0}\right\|^{2}
$$

Более того, отображение $t \mapsto V^{t}$ имеет свойство мультипликативного представления $V^{r} V^{t}=V^{r+t}$ на груше $\mathbb{R} \ni r, t$, поскольку отображение $t \mapsto S^{\Delta_{0}^{t}(s)}$ является мультипликативньм сддиговым коциклом

$$
S^{\Delta_{0}^{r}(s)} e^{t \partial_{s}} S^{\Delta_{0}^{t}(s)}=e^{t \partial_{s}} S^{\Delta_{0}^{r+t}(s)} \quad \forall r, t \in \mathbb{R}
$$

в силу аддитивности коциклического свойства для коммутирующих операторов $\Delta_{0}^{t}(s)=$ $1_{t}(s)-1_{0}(s)$ :

$$
\left[\Delta_{0}^{r}+e^{t \partial_{s}} \Delta_{0}^{t}\right](s)=1_{r}(s)-1_{0}(s)+1_{t}(s+t)-1_{0}(s+t)=e^{t \partial_{s}} \Delta_{0}^{r+t}(s) .
$$


Исключая из эволюционного оператора $\chi(t, z)=e^{\frac{i}{\hbar} t \hat{h}} \chi^{t}(z)$ генератор свободной эволюции $\hat{h} \chi(z)=i \hbar \partial_{z} \chi(z)$, получаем

$$
\begin{aligned}
\chi(t, s) & =\chi^{t}(s-t)=e^{\frac{i}{\hbar}(s-t) H} \chi_{t}(s) \\
& =e^{\frac{i}{\hbar}(s-t) H} S^{\Delta_{0}^{t}(s)} e^{-\frac{i}{\hbar} s H} \chi^{0}=V(t, s) \chi^{0}(s) .
\end{aligned}
$$

Таким образом, унитарньй коцикл $e^{-\frac{i}{\hbar} t \hat{h}}$ с разрьвом в одной точке

$$
V(t, s)=e^{\frac{i}{\hbar} t \hat{h}} V^{t}=e^{\frac{i}{\hbar}(s-t) H} S^{\Delta_{0}^{t}(s)} e^{-\frac{i}{\hbar} s H}, \quad t \in \mathbb{R},
$$

c $\Delta_{0}^{t}(s)=1_{[0, t)}(s)$ для положительных $t$ и $s \in \mathbb{R}^{+}$действительно дает решение уравнения Ито (2.2), порождаемое процессом числа частиц. Оно описывает представление взаимодействия для сильно непрерьвной унитарной группы $V^{t}$, являющейся решением задачи (2.4) с постоянной начальной функцией $\chi^{0}(s)=\eta$ при $s>0$.

ЗАмЕчаниЕ 1. Простейшая краевая задача Шрёдингера (2.4) является нефизической по трем причинам. Во-первых, уравнение (2.4) не инвариантно относительно обращения времени, т.е. относительно изометрического комплексного сопряжения $\eta \mapsto \bar{\eta}$ и отражения $t \mapsto-t$, даже если $\bar{S}=S^{-1}$ и $\operatorname{Im} H=0$. Во-вторых, физическая волновая функция должна иметь непрерывньй пропагатор в обоих направлениях $z$ и на границе должна иметь скачок не в координатном, а в импульсном представлении. Импульс может изменять направление, но не величину (закон сохранения момента) в результате сингулярного взаимодействия на границе. И в-третьих, свободньй гамильтониан $\hat{h}$ должен быть ограничен снизу, что не вьполняется в случае гамильтониана $h(z, p)=-p$, соответствующего уравнению $(2.4)^{2}$.

Сейчас мы покажем, как избавиться от первых двух недостатков простейшей модели, а третий, являющийся наиболее серьезным, мы отложим до следующего пункта, где описанная выше простейшая модель рассматривается как предельньй случай.

Вместо одной волновой функции $\chi^{t}(z)$ на $\mathbb{R}$ мы рассмотрим пару $(\psi, \tilde{\psi})$, состоящую из падающей и отраженной волн

$$
\psi^{t}(z)=\chi^{t}(z), \quad z \geqslant 0, \quad \tilde{\psi}^{t}(-z)=\chi^{t}(z), \quad z<0,
$$

на полупрямой $\mathbb{R}^{+}$со скалярным произведением

$$
\int_{0}^{\infty}\left(\|\psi(z)\|^{2}+\|\tilde{\psi}(z)\|^{2}\right) \rho(z) d z=\int_{-\infty}^{\infty}\|\chi(z)\|^{2} \rho(z) d z .
$$

Они удовлетворяют системе уравнений

$$
\begin{array}{ll}
i \hbar \partial_{t} \psi^{t}(z)=\left(H+i \hbar \partial_{z}\right) \psi^{t}(z), & \psi^{0} \in \mathscr{H} \otimes L^{2}\left(\mathbb{R}^{+}\right), \\
i \hbar \partial_{t} \tilde{\psi}^{t}(z)=\left(H-i \hbar \partial_{z}\right) \tilde{\psi}^{t}(z), & \tilde{\psi}^{0} \in \mathscr{H} \otimes L^{2}\left(\mathbb{R}^{+}\right),
\end{array}
$$

для квантовой системы, взаимодействующей в точке $z=0$ с безмассовыми частицами Дирака на $\mathbb{R}^{+}: \tilde{\psi}^{t}(0)=S \psi^{t}(0)$, где $\tilde{\psi}^{t}(0)=\chi^{t}\left(0_{-}\right)$. Нетрудно убедиться, что это

\footnotetext{
${ }^{2} \mathrm{~B}$ действительности непрерьвньм должен быть ток плотности вероятности. Кроме того, физические гамильтонианы не обязаны быть ограниченньми снизу; наиболее известными примером является матричный оператор Дирака. - Прим. переводчика.
} 
действительно диагональная форма одномерного уравнения Дирака в представлении скорости Дирака $c=-\sigma_{z}$ вдоль оси $z \in \mathbb{R}^{+}$с электрическим и магнитным потенциалами $u_{ \pm}$, заданными симметрической и антисимметрической частями $u \pm \tilde{u}$ ( $u$ на $\mathbb{R}$ в случае $\operatorname{Im} u=0)$. Компоненты $(\psi, \tilde{\psi})$ распространяются независимо вдоль $z>0$ как плоские волны в противоположных направлениях со спином, ориентированным в направлении $z$. В скалярном случае $\mathscr{H}=\mathbb{C}$ функции $(\psi, \tilde{\psi})$ связаны краевым условием дираковского типа $(1+i \mu) \tilde{\psi}^{t}(0)=(1-i \mu) \psi^{t}(0)$, соответствующего $\hbar \mu$ при $z=0$. Приходящая волна

$$
\psi^{t}(z)=e^{-\frac{i}{\hbar} t H} \psi^{0}(z+t)=e^{-\frac{i}{\hbar} t(\hat{h}+H)} \psi^{0}(z)
$$

является решением уравнения (2.4) при $z \in \mathbb{R}^{+}$с условием $\left.\chi^{0}\right|_{z>0}=\psi^{0}$, которое не требует дополнительного краевого условия в точке $z=0$ для решения задачи Коши при $t>0$. Отраженная волна удовлетворяет "отраженному уравнению" при $z>0$ и унитарному краевому условию в точке $z=0$ :

$$
i \hbar \partial_{t} \tilde{\psi}^{t}(z)=\left(H-i \hbar \partial_{z}\right) \tilde{\psi}^{t}(z), \quad \tilde{\psi}^{t}(0)=S \psi^{t}(0) .
$$

Задача (2.8) имеет решение

$$
\tilde{\psi}^{t}(z)=e^{-\frac{i}{\hbar} t H}\left[\tilde{\psi}^{0}(z-t) 1_{t}^{\perp}(z)+S(t-z) \psi^{0}(t-z) 1_{t}(z)\right],
$$

где $1_{t}^{\perp}(z)=1-1_{t}(z)$. Решение может быть записано аналогично $\psi^{t}$ в виде

$$
\tilde{\psi}^{t}(z)=e^{-\frac{i}{\hbar} t H} \tilde{\psi}^{0}(z-t)=e^{-\frac{i}{\hbar} t(\check{h}+H)} \tilde{\psi}^{0}(z),
$$

где $\breve{h}=-i \hbar \partial_{z}$, если $\psi^{0}(z)$ продолжается в область $z<0$ следуюшим образом:

$$
S(z) \psi^{t}(z)=\tilde{\psi}^{t}(-z), \quad S(z)=e^{\frac{i}{\hbar} z H} S e^{-\frac{i}{\hbar} z H}
$$

при $t=0$. Заметим, что условие отражения (2.10) остается справедливым при всех $t>0$, если $\psi^{t}$ продолжена в область $z<0$ согласно $(2.7)$ для всех $t \in \mathbb{R}^{+}$:

$$
\tilde{\psi}^{t}(-z)=e^{-\frac{i}{\hbar} t H} S(t+z) \psi^{0}(t+z)=S(z) e^{-\frac{i}{\hbar} t H} \psi^{0}(z+t)=S(z) \psi^{t}(z) .
$$

Продолжая отраженную волну $\tilde{\psi}^{t}$ согласно $(2.9)$ в область $z<0$, мы получаем непрерьвные продолжения $\psi, \tilde{\psi}$ через границу в противоположном направлении с унитарным отражением, заданньм связью (2.10) для всех $z \in \mathbb{R}$. Если $\bar{H}=H$, где $\bar{H} \eta=\bar{H} \bar{\eta}$ относительно комплексного сопряжения в $\mathscr{H}$, то система уравнений Шрёдингера для пары $(\psi, \tilde{\psi})$ оказывается инвариантной относительно обращения времени с комплексным сопряжением $\bar{\psi}^{-t} \rightleftarrows \tilde{\psi}^{t}$. Действительно, в этом случае комплексно сопряженньй гамильтониан $\overline{\hat{h}}=-i \hbar \partial_{z}$ совпадает с оператором $\check{h}$, соответствующим $\tilde{h}(z, p)=p=\bar{h}(z, p)$. Краевая задача инвариантна относительно обращения времени, если $\bar{S}=S^{-1}$, так как условие отражения (2.10) продолжается в область отрицательных $t$ путем замены $S(z)^{-1}=\bar{S}(-z)$. Таким образом, обращение времени эквивалентно перестановке приходящей и отраженной волн, являюшейся инволютивным изоморфизмом

$$
\int_{-\infty}^{\infty}\|\psi(z)\|^{2} d z=\|\chi\|^{2}=\int_{-\infty}^{\infty}\|\tilde{\psi}(z)\|^{2} d z .
$$


3. Модель унитарного отражения. Как мы видели в конце предыдущего пункта, унитарньй скачок квантового состояния в случайньй момент времени $s \geqslant 0$ является результатом решения простейшей краевой задачи для уравнения Шрёдингера в представлении взаимодействия для сильно непрерывной унитарной эволюции безмассовой частищы Дирака. Приходящая частища - "инстантон" - с вектором состояния, определяющим начальную вероятность при $s=z$, имеет неограниченную снизу кинетическую энергию $e(p)=-p$, соответствующую постоянной отрицательной скорости $v=e^{\prime}(p)=-1$ вдоль оси "внутреннего" времени $z$, которое не совпадает с направлением импульса, если $p>0$. Эту странную частищу можно интерпретировать как триггер непрерьвного измерения квантовой системы в момент $z \in \mathbb{R}^{+}$. Ее возможно рассматривать как обычную частицу с положительной кинетической энергией и ненулевой массой в пузырьковой камере на границе, как это было сделано в [2].

Наша цель состоит в получении инстантона в ультрарелятивистском пределе квантовых частиц с положительной кинетической энергией, соответствующей массе $m_{0} \geqslant 0$. Мы будем рассматривать кинетическую энергию раздельно для приходящего и уходящего инстантона как функцию момента $p \in \mathbb{R}^{-}$и $p \in \mathbb{R}^{+}$соответственно вдоль координаты $z \in \mathbb{R}^{+}$с тем же самосопряженным оператором, отображающим $e(p) \geqslant 0$ в гильбертово пространство $\mathfrak{h}$ спина или других степеней свободы. Как пример можно взять релятивистскую функцию массы

$$
e(p)=\left(p^{2}+\hbar^{2} \mu^{2}\right)^{1 / 2}, \quad \mu^{2}=\mu_{0}^{2}-\nabla^{2},
$$

в гильбертовом пространстве $\mathfrak{h}=L^{2}\left(\mathbb{R}^{d}\right)$, которая определяет скорости $v(p)=p / e(p)$ $=e^{\prime}(p)$ с тем жезнаком, что и $p$. На границе $z=0$ приходящая частица с отрицательным импульсом $p<0$ отражается с противоположным импульсом $-p$. Сингулярное взаимодействие на границе вызьвает квантовьй скачок других квантовых степеней свободы, который описывается унитарньм оператором $\sigma$, действующим в $\mathfrak{h}$. Этот оператор предполагается коммутирующим с $e(p)$ для каждого $p$, как это имеет место в модели квантовых измерений [2] с $\sigma=e^{\boldsymbol{x} \partial_{\boldsymbol{y}}}$, где $\nabla=\partial_{\boldsymbol{y}}$ в (3.1).

Пусть $\mathfrak{h}$ является гильбертовьм пространством с изометрическим комплексным сопряжением $\mathfrak{h} \ni \eta \mapsto \bar{\eta} \in \mathfrak{h}$ и $L_{\mathfrak{h}}^{2}\left(\mathbb{R}^{-}\right)=\mathfrak{h} \otimes L^{2}\left(\mathbb{R}^{-}\right)$является пространством квадратично интегрируемых функций $f(k) \in \mathfrak{h}$ на полупрямой $\mathbb{R}^{-} \ni k$. Обозначим через $\mathscr{E}^{-}$ изоморфное пространство Фурье-образов

$$
\varphi(z)=\frac{1}{2 \pi} \int_{-\infty}^{0} e^{i k z} f(k) d k, \quad f \in L_{\mathfrak{h}}^{2}\left(\mathbb{R}^{-}\right),
$$

которое является классом Харди $\mathfrak{h}$-значных функций $\varphi \in L_{\mathfrak{h}}^{2}(\mathbb{R})$, имеющих аналитическое продолжение в область $\operatorname{Im} z<0$. Пространство $L_{\mathfrak{h}}^{2}\left(\mathbb{R}^{-}\right)$можно интерпретировать как гильбертово пространство квантовых входных состояний с отрицательньм импуль$\operatorname{com} p_{k}=\hbar k, k<0$, вдоль $z \in \mathbb{R}$ и спиновыми состояниями $\eta \in \mathfrak{h}$. Обобщенные собственные функции

$$
\varphi_{k}(z)=\exp [i k z] \eta_{k}, \quad k<0, \quad e(\hbar k) \eta_{k}=\hbar \varepsilon_{k} \eta_{k}
$$

соответствующие спектральным значениям $\varepsilon_{k} \in \mathbb{R}^{+}, \varepsilon(k)=\hbar^{-1} e(\hbar k)$, имеют вид волн, приходящих из бесконечности в точку $z=0$ с фазовой скоростью $\varsigma_{k}=\varepsilon_{k} /|k|$ вдоль $z$. Амплитуды $\eta_{k}$ произвольны в $\mathfrak{h}$, если все $e(p)$ пропорциональны единичному оператору 1 из $\mathfrak{h}, \varepsilon(k)=\varepsilon_{k} 1$, как это было в предыдущем пункте, где $\mathfrak{h}=\mathscr{H}$. 
Сингулярное взаимодействие создает выходное состояние в той же области наблюдения $z>0$, где входное поле в силу инверсии импульса $p=-p_{k} \mapsto \tilde{p}=p_{k}$ отражает входную волновую функцию $\varphi \in \mathscr{E}^{-}$изометрически в

$$
\widetilde{\varphi}(s)=\frac{1}{2 \pi} \int_{-\infty}^{0} e^{-i k s} \tilde{f}(k) d k=\sigma \varphi(-s), \quad s \in \mathbb{R}_{+},
$$

поскольку $\tilde{f}(k)=\sigma f(k), k<0$. Пространство $\mathscr{E}^{+}=\left\{\widetilde{\varphi}: \varphi \in \mathscr{E}^{-}\right\}$является сопряженным классом Харди $\mathscr{E}^{+}=\left\{\bar{\varphi}: \varphi \in \mathscr{E}^{-}\right\}$аналитических функций $\bar{\varphi}(z)=\overline{\varphi(\bar{z})}$ переменной $z: \operatorname{Im} z>0$. Отраженная волна удовлетворяет краевому условию $\widetilde{\varphi}(0)=\sigma \varphi(0)$, соответствуюшему

$$
j(z)=\|\widetilde{\varphi}(z)\|^{2}-\|\varphi(z)\|^{2}
$$

в точке $z=0$, и вместе с приходящей волной $\varphi(s), s \geqslant 0$, дает квадрат гильбертовой нормы (полную вероятность) в $\mathscr{E}^{-}$и $\mathscr{E}^{+}$в виде суммы интегралов по полупространствам $\mathbb{R}_{+}$:

$$
\int_{-\infty}^{\infty}\|\varphi(z)\|^{2} d z=\int_{0}^{\infty}\left(\|\varphi(s)\|^{2}+\|\widetilde{\varphi}(s)\|^{2}\right) d s=\int_{-\infty}^{\infty}\|\widetilde{\varphi}(z)\|^{2} d z
$$

Свободная эволюция приходящей и уходящей волн описьвается унитарным пропагатоpom

$$
\begin{aligned}
& \varphi^{t}(z)=\frac{1}{2 \pi} \int_{-\infty}^{0} e^{i k(t \varsigma(k)+z)} f(k) d k=\left[e^{-i t \widehat{\varepsilon}} \varphi\right](z), \\
& \widetilde{\varphi}^{t}(z)=\frac{1}{2 \pi} \int_{-\infty}^{0} e^{i k(t \varsigma(k)-z)} \tilde{f}(k) d k=\left[e^{-i t \check{\varepsilon}} \widetilde{\varphi}\right](z)
\end{aligned}
$$

на суперпозиции гармонических собственных функций (3.2) в отрицательном и положительном направлениях оси $z \in \mathbb{R}$ соответственно с фазовыми скоростями $\varsigma_{k}>1$, которые являются собственными значениями положительных операторов $\varsigma(k)=|k|^{-1} \varepsilon(k)$. Порождающие самосопряженные операторы $\widehat{\varepsilon}, \check{\varepsilon}$, задаваемые формулами $\widehat{\varepsilon}=\varepsilon\left(i \partial_{z}\right) \mid \mathscr{D}^{-}$, $\check{\varepsilon}=\varepsilon\left(i \partial_{z}\right) \mid \mathscr{D}^{+}$, являются сужениями оператора кинетической энергии, заданными симметричной функцией $\varepsilon(p)$ на плотной симметричной области определения $\mathscr{D} \subseteq L_{\mathfrak{h}}^{2}(\mathbb{R})$, действующими в плотную область $\mathscr{D}^{\mp}=\mathscr{D} \cap \mathscr{E}^{\mp}$ в инвариантных подпространствах $\mathscr{E} \mp \subseteq L_{\mathfrak{h}}^{2}(\mathbb{R})$

Вместо рассмотрения свободного распространения пары приходящей и уходящей волн $(\varphi, \widetilde{\varphi})$ в области $z>0$ с краевым условием $\widetilde{\varphi}^{t}(0)=\sigma \varphi^{t}(0)$ удобно ввести единую волновую функцию

$$
\phi^{t}(z)=\varphi^{t}(z), \quad \operatorname{Re} z \geqslant 0, \quad \phi^{t}(z)=\tilde{\varphi}^{t}(-z), \quad \operatorname{Re} z<0
$$

рассматривая отраженную волну как волну, распространяющуюся в отрицательном направлении в области $z<0$. Каждая функция $\phi(-z)$ является функцией класса Харди $\sigma \varphi$ при $z>0$ так же, как и функция $\varphi(-z)$ при $z>0$. Непрерьвность аналитических волновых функций $\varphi(z)$ в точке $\operatorname{Re} z=0$ соответствует скачку слева $\phi\left(0_{-}\right)=\sigma \phi(0)$, т.е.

$$
\phi(z)=1_{0}(-z) \varphi(z)+1_{0}(z) \sigma \varphi(z), \quad 1_{0}(z)=\left\{\begin{array}{l}
0, z \geqslant 0 \\
1, z<0
\end{array}\right.
$$


где $\phi\left(0_{-}\right)$определяется как левьй нижний секториальньй предел функции $\phi(z)$ при $\operatorname{Re} z \nearrow 0, \operatorname{Im} z \nearrow 0$. Очевидно, что гильбертово подпространство $\sigma^{\hat{1}_{0}} \mathscr{E}-\subset L_{\mathfrak{h}}^{2}(\mathbb{R})$ таких функций изоморфно отображается в $\mathscr{E}-$ унитарным оператором $\sigma^{\hat{1}_{0}}=I+\hat{1}_{0}(\sigma-I)$, где $\hat{1}_{0}$ - оператор умножения $\varphi(z)$ на 1 , если $z<0$, и на 0 , если $z \geqslant 0$. Унитарная группа $v^{t}=\sigma^{\hat{1}_{0}} e^{-i t \widehat{\varepsilon}} \sigma^{-\hat{1}_{0}}, t \in \mathbb{R}$, для функций

$$
\phi^{t}(z)=\varphi^{t}(z)+1_{0}(z)(\sigma-1) \varphi^{t}(z)=\sigma^{1_{0}(z)} \varphi^{t}(z)
$$

унитарно эквивалентна, но отличается от свободного пропагатора $e^{-i t \widehat{\varepsilon}} \varphi^{t}$ в $\mathbb{R}$. Каждая гармоническая собственная функция (3.2), распространяющаяся как плоская волна

$$
\varphi_{k}^{t}(z)=e^{-i \varepsilon_{k} t} \varphi_{k}(z)=\varphi_{k}\left(z+\varsigma_{k} t\right)
$$

для отрицательных $k \in \mathbb{R}^{-}$, отражается $\phi_{k}(z)=e^{i k z} \sigma^{1_{0}(z)} \eta_{k}$ и распространяется в отрицательном направлении

$$
\phi_{k}^{t}(z)=\sigma^{1_{0}(z)} \varphi_{k}\left(z+\varsigma_{k} t\right)=e^{-i \varepsilon_{k} t} \phi_{k}(z) \neq \phi_{k}\left(z+\varsigma_{k} t\right)
$$

совершая поворот при $z=0$. Поэтому исключение эволюции $\phi_{t}(z)=e^{i t \widehat{\varepsilon}} \phi^{t}(z)$ из свободной эволюции $\varphi^{t}$ возврашает ее не в начальное состояние $\phi^{0}=\sigma^{\hat{1}_{0}} \varphi^{0}$, а в состояние $\phi_{t}=\sigma^{\hat{\pi}^{t}} \varphi^{0}=v_{t} \phi_{0}$, где $\hat{\pi}^{t}=e^{i t \widehat{\varepsilon} \hat{1}_{0}} e^{-i t \widehat{\varepsilon}}, v_{t}=\sigma^{\hat{\pi}^{t}} \sigma^{-\hat{1}_{0}}$ и $\phi_{0}=\phi^{0}$. Таким образом, мы доказали следующее утверждение для специального случая $\varkappa=0$ оператора $\varkappa \in \mathbb{R}$, определенного в предложении 1 предыдущего пункта как $\hbar \varkappa=H$ в $\mathfrak{h}=\mathscr{H}$.

Пусть $\varkappa$ - самосопряженньй оператор в $\mathfrak{h}$ и $\epsilon_{\varkappa}(z)=e^{-i \varkappa z}-$ соответствующая однопараметрическая унитарная группа в $\mathfrak{h}$. Ниже мы обозначаем через $\hat{\epsilon}_{\varkappa}$ и $\check{\epsilon}_{\varkappa}$ операторы поточечного умножения на функции $\epsilon_{\varkappa}: z \mapsto \epsilon_{\varkappa}(z)$ и $\widetilde{\epsilon_{\varkappa}}: z \mapsto \epsilon_{\varkappa}(-z)$ от $z \in \mathbb{R}$ соответственно. Оба эти оператора унитарны в гильбертовом пространстве $L_{\mathfrak{h}}^{2}(\mathbb{R})$. Если $\hbar \hat{\gamma}=\hat{h}$ является оператором в $L_{\mathfrak{h}}^{2}(\mathbb{R})$, который задается псевдодифференциальным оператором $h\left(z, \frac{\hbar}{i} \partial_{z}\right)=\hbar \gamma\left(z, i \partial_{z}\right)$, то операторная функция

$$
\gamma(z, \kappa+\varkappa)=\epsilon_{\varkappa+\kappa}^{*}(z) \gamma\left(z, i \partial_{z}\right) \epsilon_{\varkappa+\kappa}(z) \equiv \gamma_{\varkappa+\kappa}(z)
$$

определяет символ $\gamma_{\varkappa}(z, \kappa)=\gamma(z, \varkappa+\kappa)$ оператора

$$
\hat{\gamma}_{\varkappa}=\hat{\epsilon}_{\varkappa}^{*} \hat{\gamma} \hat{\epsilon}_{\varkappa} \equiv \gamma_{\varkappa}\left(z, i \partial_{z}\right)
$$

Он определен на экспоненциальных функциях $\epsilon_{\kappa}(z)=e^{-i \kappa z}$ как псевдодифференциальный оператор

$$
\left[\hat{\gamma}_{\varkappa} \epsilon_{\kappa} \eta\right](z)=\gamma_{\varkappa}\left(z, i \partial_{z}\right) e^{-i \kappa z} \eta=e^{-i \kappa z} \gamma_{\varkappa}(z, \kappa) \eta, \quad \eta \in \mathfrak{h}
$$


УТВЕРЖДЕНИЕ 3.1. Пусть $\mathscr{E}_{0}^{-}=\mathscr{E}^{-}-$класс Харди пространства $L_{\mathfrak{h}}^{2}(\mathbb{R}), \mathscr{E}_{\varkappa}-\subset$ $L_{\mathfrak{h}}^{2}(\mathbb{R})$ - гильбертово пространство функиий $\varphi=\hat{\epsilon}_{\varkappa}^{*} \varphi_{0} c \varphi_{0} \in \mathscr{E}_{0}^{-}, \mathscr{E}_{\varkappa}^{+}=\check{\epsilon}_{\varkappa}^{*} \mathscr{E}_{0}^{+}$, əде $\mathscr{E}_{0}^{+}=\mathscr{E}^{+}$. Пусть начальная краевая задача для уравнения Шрёдингера

$$
\begin{aligned}
i \partial_{t} \varphi^{t}(z) & =\varepsilon_{\varkappa}\left(i \partial_{z}\right) \varphi^{t}(z), & & \varphi^{0} \in \mathscr{E}_{\varkappa}^{-}, \quad z>0, \\
i \partial_{t} \widetilde{\varphi}^{t}(z) & =\tilde{\varepsilon}_{\varkappa}\left(i \partial_{z}\right) \widetilde{\varphi}^{t}(z), & & \widetilde{\varphi}^{t}(0)=\sigma \varphi^{t}(0),
\end{aligned}
$$

определена генераторами $\hat{\varepsilon}_{\varkappa}, \check{\varepsilon}_{\varkappa}$, заданными символами

$$
\varepsilon_{\varkappa}(\kappa)=\varepsilon(\varkappa+\kappa), \quad \tilde{\varepsilon}_{\varkappa}(\kappa)=\varepsilon(\varkappa-\kappa)
$$

соответственно, где $\varepsilon(\kappa)$ - симметричная функиия от $\kappa \in \mathbb{R}$, соответствующая кинетической энергии $е(p)=\hbar \varepsilon\left(\hbar^{-1} p\right)>0$. Тогда задача (3.7) самосопряжена, если исходные и уходящие волны $\widetilde{\varphi}^{0}$ определены в $\mathscr{E}_{\varkappa}^{+}$соотношением $\widetilde{\varphi}^{0}(-z)=\sigma_{\varkappa}(z) \varphi^{0}(z), z<0$, әде $\sigma_{\varkappa}=\epsilon_{\varkappa}^{*} \sigma \epsilon_{\varkappa}$, по аналитическому продолжению функиии $\varphi_{0}^{0}=\hat{\epsilon}_{\varkappa} \varphi^{0}$ в область $\mathbb{R}^{-}$. Решения уравнения (3.7) можно записать в виде

$$
\varphi^{t}(z)=\phi^{t}(z), \quad z \geqslant 0, \quad \widetilde{\varphi}^{t}(-z)=\phi^{t}\left(z_{-}\right), \quad z \leqslant 0,
$$

əде $\phi^{t}=e^{-i t \hat{\varepsilon}_{\varkappa}} \phi_{t}, \phi_{t}=\varphi^{0}+\left(1-\hat{\sigma}_{\varkappa}\right) \hat{\pi}_{\varkappa}^{t} \varphi^{0}, \hat{\sigma}_{\varkappa}$ является поточечным умножением на $\sigma_{\varkappa}(z) u$

$$
\hat{\pi}_{\varkappa}^{t}=e^{i t \hat{\varepsilon}_{\varkappa}} \hat{1}_{0} e^{-i t \hat{\varepsilon}_{\varkappa}} \equiv \pi_{\varkappa}^{t}\left(z, i \partial_{z}\right)
$$

задано символом $\pi^{t}(z, \kappa)$ ортопроектора $\hat{\pi}^{t}=e^{i t \hat{\varepsilon}} \hat{1}_{0} e^{-i t \hat{\varepsilon}}$ как и в (3.6).

ДокАЗАтЕльСтво. Пусть $\varphi^{t}=e^{-i \varepsilon_{k} t} \varphi_{k}, \widetilde{\varphi}^{t}=e^{-i \varepsilon_{k} t} \widetilde{\varphi}_{k}$. Рассмотрим стационарное уравнение Шрёдингера

$$
\varepsilon_{\varkappa}\left(z, i \partial_{z}\right) \varphi_{k}(z)=\varepsilon_{k} \varphi_{k}(z), \quad \widetilde{\varphi}_{k}(-z)=\sigma_{\varkappa}(z) \varphi_{k}(z),
$$

соответствующее заданным в (3.7) начальному условию и краевым условиям. Здесь $\varphi_{k}$ продолжены в область $\mathbb{R}^{-}$с помошью аналитического продолжения функций $\epsilon_{\varkappa}^{*} \varphi_{k}$, которые являются обобщенными собственными функциями (3.2) оператора $\hat{\varepsilon}=\varepsilon\left(i \partial_{z}\right)$ в $\mathscr{E}_{0}^{-}$тогда и только тогда, когда $k<0$. В силу самосопряженности $\hat{\varepsilon}$ в $\mathscr{E}-$ собственные функции $\varphi_{k}=\epsilon_{\varkappa+k}^{*} \eta_{k}$ оператора $\hat{\varepsilon}_{\varkappa}$ для (3.9) с отрицательньми $k$ образуют ортогональное дополнение гильбертова пространства $\mathscr{E}-$, и уходящие собственные функции $\widetilde{\varphi}_{k}(z)=\widetilde{\epsilon_{\varkappa+k}^{*}}(z) \tilde{\eta}_{k}, \sigma_{0}=\rho_{0}^{-1 / 2} \sigma \rho_{0}^{1 / 2}$ образуют ортогональное дополнение гильбертова пространства $\mathscr{E}_{\varkappa}^{+}$, где $\tilde{\eta}_{k}=\sigma_{0} \eta_{k}$. Решения уравнений (3.7) могут быть записаны следующим образом в виде (3.3):

$$
\begin{aligned}
& \varphi^{t}(z)=\frac{1}{2 \pi} \int_{-\infty}^{0} e^{-i \varepsilon(k) t} \epsilon_{\varkappa+k}^{*}(z) f(k) d k=\left[e^{-i t \hat{\varepsilon}_{\varkappa}} \varphi^{0}\right](z), \\
& \widetilde{\varphi}^{t}(z)=\frac{1}{2 \pi} \int_{-\infty}^{0} e^{-i \varepsilon(k) t} \widetilde{\epsilon_{\varkappa+k}^{*}}(z) \tilde{f}(k) d k=\left[e^{-i t \tilde{\varepsilon}_{\varkappa}} \widetilde{\varphi}^{0}\right](z),
\end{aligned}
$$

где $\tilde{f}_{0}(k)=\sigma f_{0}(k)$ определены как преобразования Фурье

$$
f(k)=\int_{-\infty}^{\infty} \epsilon_{\varkappa+k}(z) \varphi^{0}(z) d z, \quad \tilde{f}(k)=\int_{-\infty}^{\infty} \widetilde{\epsilon_{\varkappa+k}}(z) \widetilde{\varphi}^{0}(z) d z
$$


а начальные условия аналитически продолжены на всю прямую $\mathbb{R}$. В силу коммутативности $\sigma$ и $\hat{\varepsilon}$ они удовлетворяют соотношению $\tilde{\varphi}^{t}(-z)=\sigma_{\varkappa}(z) \varphi^{t}(z)$ для всех $t$, а не только для $t=0$. Инвариантность этого соотношения и унитарность группы преобразований, действующей в гильбертовом пространстве $\mathscr{E}-\oplus \mathscr{E}_{\varkappa}^{+}$, следующие из унитарности $(3.3)$ в $\mathscr{E} \mp \subset L_{\mathfrak{h}}^{2}(\mathbb{R})$, означают самосопряженность задачи (3.7) для пары $\varphi^{\mp} \in L_{\mathfrak{h}}^{2}(\mathbb{R}, \rho)$ в области определения оператора $\hat{\varepsilon}_{\varkappa} \oplus \check{\varepsilon}_{\varkappa}$ с условием $\varphi^{+}(-z)=\sigma_{\varkappa}(z) \varphi^{-}(z)$. Вводя в рассмотрение

$$
\phi^{t}(z)=\varphi^{t}(z)+1_{0}(z)\left(\sigma_{\varkappa}(z)-1\right) \varphi^{t}(z)=\sigma_{\varkappa}(z)^{1_{0}(z)} \varphi^{t}(z),
$$

как и в случае (3.5), и принимая во внимание, что

$$
\phi^{t}\left(z_{-}\right)=\sigma_{\varkappa}(z)^{1_{0}\left(z_{-}\right)} \varphi^{t}(z)=\sigma_{\varkappa}(z)^{1-1_{0}(-z)} \varphi^{t}(z)=\sigma_{\varkappa}(z)^{-1_{0}(-z)} \widetilde{\varphi}^{t}(-z),
$$

мы получаем, что представление $(3.8)$ для $\varphi^{t}(z)$ совпадает с $\phi^{t}(z)$ при $z \geqslant 0$ и для $\widetilde{\varphi}^{t}(-z)$ с $\tilde{\phi}^{t}(-z)=\phi^{t}\left(z_{-}\right)$при $z \leqslant 0$.

ЗАмечАниЕ 2. Краевая задача (3.7) для уравнения Шрёдингера является физической во всех трех аспектах. Во-первых, уравнение (3.7) инвариантно относительно направления времени, т.е. относительно отражения $t \mapsto-t$ и изометрического сопряжения $\varphi \mapsto \bar{\varphi}$ вместе с перестановкой приходящей и уходящей волн $\varphi \leftrightarrows \widetilde{\varphi}$, если $\bar{\sigma}=\sigma^{-1}$ и $\bar{\varkappa}=\tilde{\varkappa}$, где $\tilde{\varkappa}(z)=\varkappa(-z)$. Во-вторых, волновая функция $\varphi^{t}, \tilde{\varphi}^{t}$ описывает непрерьвное распространение импульса вдоль $z$. При $z=0$ импульс меняет направление, но не величину, в результате действия краевого условия $\varphi(0) \mapsto \widetilde{\varphi}(0)$. И в-третьих, кинетическая энергия $\hat{\varepsilon}_{\varkappa} \oplus \check{\varepsilon}_{\varkappa}$ ограничена снизу в силу унитарного преобразования $\hat{\varepsilon} \simeq \check{\varepsilon}(2.4)$.

Действительно, из $\bar{\varepsilon}(\kappa)=\varepsilon(\kappa)=\widetilde{\varepsilon}(\kappa)$ следует, что символ $\bar{\varepsilon}_{\varkappa}(z, \kappa)=\overline{\varepsilon_{\varkappa-\kappa}}(z)$ комплексно сопряженного оператора $\overline{\varepsilon_{\varkappa}}$ имеет вид

$$
\overline{\varepsilon_{\varkappa}}(z)=\epsilon_{-\bar{\varkappa}}^{*}(z) \varepsilon\left(i \partial_{z}\right) \epsilon_{-\bar{\varkappa}}(z)=\epsilon_{-\tilde{\varkappa}}^{*}(z) \varepsilon\left(i \partial_{z}\right) \epsilon_{-\tilde{\varkappa}}(z)=\widetilde{\varepsilon_{\varkappa}}(z),
$$

если $\bar{\varkappa}=\tilde{\varkappa}$, поскольку $\overline{\epsilon_{\varkappa}}(z)=\epsilon_{-\bar{\varkappa}}(z)$ и $\epsilon_{-\tilde{\varkappa}}(z)=\widetilde{\epsilon_{\varkappa}}(z)$. Поэтому $\bar{\varepsilon}_{\varkappa}(z, \kappa)=\tilde{\varepsilon}_{\varkappa}(z, \kappa)$, где $\tilde{\varepsilon}_{\varkappa}(z, \kappa)=\widetilde{\varepsilon_{\varkappa-\kappa}}(z)$, является символом оператора кинетической энергии $\check{\varepsilon}_{\varkappa}=\widetilde{\hat{\varepsilon}_{\varkappa}}$ уходящей волны $\widetilde{\varphi}$. Таким образом, обращение времени совместно с комплексным сопряжением в (3.7) эквивалентно перестановке приходящей и уходящей волн $\left(\varphi^{t}, \widetilde{\varphi}^{t}\right) \mapsto$ $\left(\widetilde{\varphi}^{t}, \varphi^{t}\right)$, которая сохраняет связь между $\varphi^{t}$ и $\widetilde{\varphi}^{t}$, поскольку

$$
\overline{\sigma_{\varkappa}}(z)=\epsilon_{-\bar{\varkappa}}^{*}(z) \bar{\sigma} \epsilon_{-\bar{\varkappa}}(z)=\epsilon_{-\tilde{\varkappa}}^{*}(z) \sigma^{-1} \epsilon_{-\tilde{\varkappa}}(z)=\widetilde{\sigma_{\varkappa}}(z)^{-1},
$$

где $\widetilde{\sigma_{\varkappa}}(z)=\sigma_{\varkappa}(-z)$ в силу $\bar{\sigma}=\sigma^{-1}$.

4. Ультрарелятивистский предел. Здесь мы предполагаем, что положительный оператор кинетической энергии $e(p)$ имеет релятивистскую форму $|p|$, или в более общем случае $e(p)=\sqrt{p^{2}+\hbar^{2} \mu^{2}}$, как было предположено в (3.1). Это соответствует конечным границам $v_{\mp}=\mp 1$ скорости $v(p)=\varepsilon^{\prime}(p)$ при $p \rightarrow \mp \infty$. Заметим, что фазовая скорость

$$
\varsigma_{\kappa}=\frac{\varepsilon(\kappa)}{\kappa}=\sqrt{1+\frac{\varepsilon^{2}}{\kappa^{2}}}=\left|v(\hbar \kappa)^{-1}\right|
$$

для импульса $p=\mp \hbar \kappa, \kappa>0$, гармонической волновой функции

$$
e^{-i \varepsilon_{\kappa} t} \epsilon_{\kappa}(z)=e^{-i \kappa\left(\varsigma_{\kappa} t+z\right)}, \quad e^{-i \varepsilon_{\kappa} t} \tilde{\epsilon}_{\kappa}(z)=e^{-i \kappa\left(\varsigma_{\kappa} t-z\right)}
$$


также имеет предел $\varsigma=1$ при $\kappa \rightarrow \infty$. Поэтому можно ожидать, что в случае быстро осциллирующих приходящих и уходящих волн

$$
\varphi^{t}(z)=e^{-i \kappa(t+z)} \psi^{t}(z), \quad \tilde{\varphi}^{t}(z)=e^{-i \kappa(t-z)} \tilde{\psi}^{t}(z),
$$

где

$$
\psi^{t}(z)=\psi(z+t) \equiv e^{t \partial_{z}} \psi, \quad \tilde{\psi}^{t}(z)=\tilde{\psi}(z-t) \equiv e^{t \tilde{\partial}_{z}} \tilde{\psi}
$$

в ультрарелятивистском пределе $p \rightarrow \mp \infty$ решения распространяются как плоскиеволны, если начальные условия приготовлены в виде волн с медленно изменяющейся амплитудой $\psi, \tilde{\psi} \in L_{\mathfrak{h}}^{2}(\mathbb{R})$. Такое распространение воспроизводит динамику отражения от гранищы $\tilde{\psi}^{t}(0)=\sigma \psi(0)$ на полупрямой $\mathbb{R}^{+} \ni z=s$, если амплитуды начальных волн связаны соотношениями $\tilde{\psi}(-z)=\sigma \psi(z)$ для всех $z \in \mathbb{R}$. В частности, решения

$$
\begin{aligned}
& \psi^{t}(s)=\psi(s+t), \quad \tilde{\psi}^{t}(s)=0, \quad t<s \\
& \tilde{\psi}^{t}(s)=\tilde{\psi}^{t-s}(0)=\sigma \psi^{t-s}(0)=\sigma \psi(t-s), \quad t>s
\end{aligned}
$$

этой гамильтоновой краевой задачи с приходящей волновой функцией

$$
\psi(z)=\sqrt{\rho(z)} \eta, \quad z>0, \quad \psi(z)=0, \quad z \leqslant 0
$$

для начального вектора состояния $\eta \in \mathfrak{h}$ будут соответствовать скачкообразной стохастической динамике в положительном направлении времени $t$ относительно плотности вероятности $\rho>0, \int_{0}^{\infty} \rho(s) d s=1$.

Ниже мы приводим точную формулировку и доказательство этой гипотезы в более общей постановке, которая необходима для вьвода квантовой стохастической эволюции как вторично квантованной краевой задачи. Но сначала мы введем обозначения и проиллюстрируем этот пример в простейшем случае.

В дальнейшем мы будем использовать понятие индуктивного предела возрастающей последовательности $\left(\mathscr{E}_{\kappa}\right)_{\kappa>0}$ гильбертовых подпространств $\mathscr{E}_{\kappa} \subseteq \mathscr{E}_{\kappa^{\prime}}, \kappa<\kappa^{\prime}$. Он определяется как объединение $\mathscr{E}=\bigcup \mathscr{E}_{\kappa}$, снабженное индуктивной сходимостью, которая совпадает с равномерной сходимостью на одном из подпространств $\mathscr{E}_{\kappa}$ и поэтому является более сильной, чем сходимость в равномерном пополнении $\mathscr{K}=\overline{\mathscr{E}}$. Двойственная индуктивная сходимость слабее, чем сходимость в $\mathscr{K}$, а индуктивная сходимость операторов в $\mathscr{E}$ определяется как сходимость операторов, действующих из каждого $\mathscr{\kappa} к$ в одно из подпространств $\mathscr{E}_{\kappa^{\prime}} \subseteq \mathscr{K}$.

Пусть $\mathscr{G}^{-}=\bigcup \mathscr{E}_{\kappa}^{-}, \mathscr{G}^{+}=\bigcup \mathscr{E}_{\kappa}^{+}$являются индуктивньми пределами для возрастающей последовательности $\left(\mathscr{E}_{\kappa}^{-}, \mathscr{E}_{\kappa}^{+}\right) \kappa>0$ классов Харди

$$
\mathscr{E}_{\kappa}^{-}=\hat{\epsilon}_{\kappa}^{*} \mathscr{E}^{-} \supset \mathscr{E}_{\kappa^{\circ}}^{-}, \quad \mathscr{E}_{\kappa}^{+}=\check{\epsilon}_{\kappa}^{*} \mathscr{E}^{+} \supset \mathscr{E}_{\kappa^{\circ}}^{+}, \quad \kappa^{\circ}<\kappa
$$

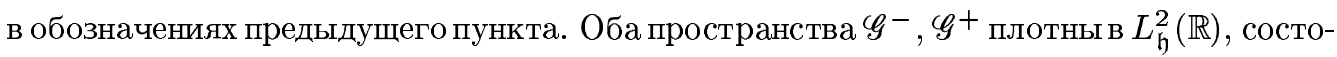
ящем из квадратично интегрируемых $\mathfrak{h}$-значных функций $\psi \in \mathscr{G}^{-}, \tilde{\psi} \in \mathscr{G}^{+}$, имеюших нулевые Фурье-образы

$$
g(k)=\int_{-\infty}^{\infty} e^{-i k z} \psi(z) d z, \quad \tilde{g}(k)=\int_{-\infty}^{\infty} e^{i k z} \tilde{\psi}(z) d z
$$


для всех $k \geqslant \kappa$ с достаточно большими $\kappa>0$. Если $\psi \in \mathscr{E}_{\kappa}^{-}$и $\tilde{\psi} \in \mathscr{E}_{\kappa}^{+}$, то $\varphi=\epsilon_{\kappa} \psi \in \mathscr{E}^{-}$, $\tilde{\varphi}=\tilde{\epsilon}_{\kappa} \tilde{\psi} \in \mathscr{E}^{+}$и свободная эволюция (3.3) может быть записана в виде (4.1), где

$$
\begin{aligned}
& \psi^{t}=e^{i \kappa t} \hat{\epsilon}_{\kappa}^{*} \varphi^{t}=\hat{\epsilon}_{\kappa}^{*} e^{-i(\hat{\varepsilon}-\kappa 1) t} \hat{\epsilon}_{\kappa} \psi \equiv \psi_{\kappa}^{t}, \\
& \tilde{\psi}^{t}=e^{i \kappa t} \check{\epsilon}_{\kappa}^{*} \tilde{\varphi}^{t}=\check{\epsilon}_{\kappa}^{*} e^{-i(\check{\varepsilon}-\kappa 1) t} \check{\epsilon}_{\kappa} \tilde{\psi} \equiv \tilde{\psi}_{\kappa}^{t} .
\end{aligned}
$$

Эти унитарные преобразования в $\mathscr{E}_{\kappa}^{-}$и в $\mathscr{E}_{\kappa}^{+}$, записанные в виде

$$
\psi_{\kappa}^{t}(z)=e^{-i t \omega_{\kappa}\left(i \partial_{z}\right)} \psi(z), \quad \tilde{\psi}_{\kappa}^{t}(z)=e^{-i t \tilde{\omega}_{\kappa}\left(i \partial_{z}\right)} \tilde{\psi}(z)
$$

порождаются самосопряженными операторами

$$
\begin{aligned}
& \omega_{\kappa}\left(i \partial_{z}\right)=e^{i \kappa z}\left(\varepsilon\left(i \partial_{z}\right)-\kappa\right) e^{-i \kappa z}=\varepsilon\left(\kappa+i \partial_{z}\right)-\kappa, \\
& \widetilde{\omega}_{\kappa}\left(i \partial_{z}\right)=e^{-i \kappa z}\left(\varepsilon\left(i \partial_{z}\right)-\kappa\right) e^{i \kappa z}=\varepsilon\left(\kappa-i \partial_{z}\right)-\kappa .
\end{aligned}
$$

Они оставляют инвариантными все подпространства $\mathscr{E}_{\kappa^{\circ}}^{-}$и $\mathscr{E}_{\kappa^{\circ}}^{+}$соответственно, хотя их генераторы $\hat{\omega}_{\kappa}, \check{\omega}_{\kappa}$ не являются положительно определенньпи при достаточно больших $\kappa$ и не являются унитарно эквивалентными для различных $\kappa$, поскольку

$$
\begin{aligned}
& \hat{\epsilon}_{\varkappa} \widehat{\omega}_{\kappa} \hat{\epsilon}_{\varkappa}^{*}=\hat{\varepsilon}_{\kappa}^{\circ}-\kappa 1=\widehat{\omega}_{\kappa^{\circ}}-\varkappa 1, \\
& \check{\epsilon}_{\varkappa} \check{\omega}_{\kappa} \check{\epsilon}_{\varkappa}^{*}=\check{\varepsilon}_{\kappa^{\circ}}-\kappa 1=\check{\omega}_{\kappa^{\circ}}-\varkappa 1,
\end{aligned}
$$

где $\varkappa=\kappa-\kappa^{\circ}$. Таким образом, мы должны доказать, что оператор (4.4) имеет индуктивньй предел в виде плоской волны (4.2) при $\kappa \rightarrow \infty$, соответствующей пределам в форме Дирака

$$
\lim _{\kappa \rightarrow \infty} \omega_{\kappa}\left(i \partial_{z}\right)=i \partial_{z}, \quad \lim _{\kappa \rightarrow \infty} \widetilde{\omega}_{\kappa}\left(i \partial_{z}\right)=-i \partial_{z}
$$

для генераторов Шрёдингера (4.4).

Следуюший факт, который мы докажем для вьвода стохастического предела, состоит в том, что отраженная волна

$$
\chi_{\kappa}^{t}=e^{-i t \hat{\omega}_{\kappa}} \chi_{\kappa, t}, \quad \chi_{\kappa, t}=\psi+(1-\sigma) \hat{\pi}_{\kappa}^{t} \psi
$$

представленная парой (4.4) на полупрямой $\mathbb{R}^{+} \ni z($ см. (3.8)), имеет разрывный предел

$$
\chi^{t}(z)=\chi_{t}(z+t), \quad \chi_{t}=\psi+(1-\sigma) \hat{1}_{t} \psi
$$

Здесь $\hat{1}_{t}=e^{-i t \partial_{z}} \hat{1}_{0} e^{i t \partial_{z}}-$ поточечное умножение на характеристическую функцию $1_{t}$ интервала $-\infty<z<t$, которую мы получим как индуктивный предел ортопроекторов

$$
\hat{\pi}_{\kappa}^{t}=e^{i t \varepsilon\left(\kappa+i \partial_{z}\right)} \hat{1}_{0} e^{-i t \varepsilon\left(\kappa+i \partial_{z}\right)}=e^{i t \hat{\omega}_{\kappa}} \hat{1}_{0} e^{-i t \hat{\omega}_{\kappa}}
$$

при $\kappa \rightarrow \infty$. Эти результаты сформулированы в следующем предложении в общем случае в обозначениях утверждения 3.1 . 
УТВЕРЖДЕНИЕ 4.1. Пусть $\mathscr{G}^{-}$- әильбертовый индуктивный предел классов Харди $\mathscr{E}_{\kappa}=\hat{\epsilon}_{\kappa}^{*} \mathscr{E}^{-}$, и пусть $\mathscr{G}_{\varkappa}^{-} \subset L_{\mathfrak{h}}^{2}(\mathbb{R}, \rho)$ - әильбертово пространство функиий $\psi=\hat{\epsilon}_{\varkappa}^{*} \psi_{0}$, əде $\psi_{0} \in \mathscr{G}^{-} \equiv \mathscr{G}_{0}^{-}$. Пусть maкжсе $\mathscr{G}_{\varkappa}^{+}=\check{\epsilon}_{\varkappa}^{*} \mathscr{G}^{+}$, əде $\mathscr{G}^{+}=\cup \mathscr{E}_{\kappa}^{+} \equiv \mathscr{G}_{0}^{+}$. Пусть начальное условие краевой задачи для уравнения Шрёдингера

$$
\begin{array}{ll}
i \partial_{t} \psi_{\kappa}^{t}(z)=\omega_{\varkappa, \kappa}\left(z, i \partial_{z}\right) \psi_{\kappa}^{t}(z), & \psi_{\kappa}^{0}=\psi \in \mathscr{G}_{\varkappa}^{-}, \quad z>0, \\
i \partial_{t} \tilde{\psi}_{\kappa}^{t}(z)=\widetilde{\omega}_{\varkappa, \kappa}\left(z, i \partial_{z}\right) \tilde{\psi}_{\kappa}^{t}(z), & z>0, \quad \tilde{\psi}_{\kappa}^{t}(0)=\sigma \tilde{\psi}_{\kappa}^{t}(0),
\end{array}
$$

определено генераторами

$$
\hat{\omega}_{\varkappa, \kappa}=\hat{\epsilon}_{\varkappa}^{*} \hat{\omega}_{\kappa} \hat{\epsilon}_{\varkappa}, \quad \check{\omega}_{\varkappa, \kappa}=\check{\epsilon}_{\varkappa}^{*} \check{\omega}_{\kappa} \check{\epsilon}_{\varkappa}
$$

с символами $\omega_{\kappa}, \widetilde{\omega}_{\kappa}$, введенными в формулах (4.6), (3.1), и начальным значением $\tilde{\psi}_{\kappa}^{0}=\tilde{\psi}$, определенным в $\mathscr{G}_{\varkappa}^{+} \kappa$ ка $\tilde{\psi}(-z)=\sigma_{\varkappa}(z) \psi(z), z<0$, с помощью аналитического продолэсения каждой функиии $\psi_{0}=\hat{\epsilon}_{\varkappa} \psi$ в область $\mathbb{R}^{-}$. Тогда решения (4.7) сходятся индуктивно к

$$
\psi^{t}(z)=\chi^{t}(z), \quad z \geqslant 0, \quad \tilde{\psi}^{t}(-z)=\chi^{t}\left(z_{-}\right), \quad z \leqslant 0,
$$

əде $\chi^{t}(z)=\epsilon_{\varkappa}(t) \chi_{t}(z+t) u \chi_{t}=\psi+\left(1-\hat{\sigma}_{\varkappa}\right) \hat{1}_{t} \psi$.

ДокАЗАТЕЛЬСтво. Прежде всего заметим, что генераторы в формуле (4.7) имеют формальные пределы

$$
\begin{aligned}
& \lim _{\kappa \rightarrow \infty}\left[\hat{\omega}_{\varkappa, \kappa} \psi\right](z)=\epsilon_{\varkappa}^{*}(z) i \partial_{z}\left[\epsilon_{\varkappa} \psi\right](z)=\left(\varkappa+i \partial_{z}\right) \psi(z), \\
& \lim _{\kappa \rightarrow \infty}\left[\check{\omega}_{\varkappa, \kappa} \tilde{\psi}\right](z)=\widetilde{\epsilon_{\varkappa}^{*}}(z) i \tilde{\partial}_{z}\left[\tilde{\epsilon}_{\varkappa} \tilde{\psi}\right](z)=\left(\varkappa+i \tilde{\partial}_{z}\right) \tilde{\psi}(z),
\end{aligned}
$$

где $\tilde{\partial}_{z}=-\partial_{z}$. Это следует из (4.6) и равенств $i \partial_{z} \epsilon_{\varkappa}=\varkappa \epsilon_{\varkappa}, \partial_{z} \widetilde{\epsilon_{\varkappa}}=i \tilde{\varkappa} \widetilde{\epsilon_{\varkappa}}$, поскольку $\widetilde{\epsilon_{\varkappa}}(z)=\epsilon_{-\varkappa}(z)$. Следовательно, мы должны доказать, что решения уравнения (4.7) имеют пределы $\psi=\lim \psi_{\kappa}, \tilde{\psi}=\lim \tilde{\psi}_{\kappa}$ в $\mathscr{G}_{\varkappa}^{\mp}$, совпадающие с решениями краевой задачи Дирака

$$
\begin{array}{ll}
i \partial_{t} \psi^{t}(z)=\left(\varkappa+i \partial_{z}\right) \psi^{t}(z), & \psi^{0}=\psi \in \mathscr{G}_{\varkappa}^{-}, \quad z>0, \\
i \partial_{t} \tilde{\psi}^{t}(z)=\left(\varkappa+i \tilde{\partial}_{z}\right) \tilde{\psi}^{t}(z), & z>0, \quad \tilde{\psi}^{t}(0)=\sigma_{0} \psi^{t}(0)
\end{array}
$$

с начальным условием $\tilde{\psi}^{0}$, заданньм аналитически формулой $\tilde{\psi}^{0}(-z)=\sigma_{\varkappa}(z) \psi^{0}(z)$, для того чтобы решение $\tilde{\psi}^{t}$ принадлежало $\mathscr{G}_{\varkappa}^{-}$для всех $t$.

Докажем это с помощю изоморфизма $\hat{\epsilon}_{\varkappa} \check{\epsilon}_{\varkappa}$ из плотных подпространств $\mathscr{G}_{\varkappa}^{\mp}$ и $\mathscr{G}_{0}^{\mp} \subset$ $L_{\mathfrak{h}}^{2}(\mathbb{R})$. В силу этого краевая задача (4.7) эквивалентна задаче

$$
\begin{aligned}
& i \partial_{t} \psi_{0, \kappa}^{t}(z)=\omega_{\kappa}\left(i \partial_{z}\right) \psi_{0, \kappa}^{t}(z), \quad \psi_{0, \kappa}^{0}=\psi_{0} \in \mathscr{G}_{0}^{-}, \quad z>0, \\
& i \partial_{t} \tilde{\psi}_{0, \kappa}^{t}(z)=\widetilde{\omega}_{\kappa}\left(i \partial_{z}\right) \tilde{\psi}_{0, \kappa}^{t}(z), \quad z>0, \quad \tilde{\psi}_{0, \kappa}^{t}(0)=\sigma_{0} \tilde{\psi}_{0, \kappa}^{t}(0),
\end{aligned}
$$

где $\omega_{\kappa}(-k)=\varepsilon(\kappa-k)-\kappa=\widetilde{\omega}_{\kappa}(k)$ и $\tilde{\psi}_{0, \kappa}^{0}(-z)=\sigma \psi_{0}(z)$, поскольку $\sigma_{\kappa}=\epsilon_{\kappa}^{*} \sigma \epsilon_{\kappa}=\sigma$ для любого скаляра $\kappa$. Таким образом, мы должны найти ультрарелятивистский предел решений

$$
\begin{aligned}
& {\left[e^{-i t \hat{\omega}_{\kappa}} \psi_{0}\right](z)=\frac{1}{2 \pi} \int_{-\infty}^{\kappa} e^{-i\left(t \omega_{\kappa}(-k)-k z\right)} g(k) d k} \\
& {\left[e^{-i t \check{\omega}_{\kappa}} \tilde{\psi}_{0}\right](z)=\frac{1}{2 \pi} \int_{-\infty}^{\kappa} e^{-i\left(t \omega_{\kappa}(-k)+k z\right)} \tilde{g}(k) d k}
\end{aligned}
$$


где $\tilde{g}(k)=\sigma g(k)$ при $\kappa \rightarrow \infty$. Здесь Фурье-образы

$$
g(k)=\int_{-\infty}^{\infty} e^{-i k z} \psi_{0}(z) d z, \quad \tilde{g}(k)=\int_{-\infty}^{\infty} e^{i k z} \tilde{\psi}_{0}(z) d z
$$

определены с помощью аналитического продолжения начального условия $\psi_{0} \in \hat{\epsilon}_{\kappa^{\circ}}^{*} \mathscr{E}_{0}^{-}$, $\tilde{\psi}_{0} \in \check{\epsilon}_{\kappa^{\circ}}^{*} \mathscr{E}_{0}^{+}$для $\kappa^{\circ}<\kappa$ таких, что интегрирование в выражениях (4.9) может быть сужено на $k<\kappa^{\circ}$, поскольку $g(k)=0=\tilde{g}(k)$ для всех $k \geqslant \kappa^{\circ}$. Поэтому доказательство того, что унитарная эволюция (4.9) индуктивно сходится к плоским волнам $e^{t \partial_{z}} \psi_{0}$, $e^{t \tilde{\partial}_{z}} \tilde{\psi}_{0}$, разрешающим эту задачу при $\kappa \rightarrow \infty$, может быть сведено к оценке интеграла

$$
I\left(\kappa^{\circ}, \kappa\right)=\frac{1}{2 \pi} \int_{-\infty}^{\kappa^{\circ}}\left\|\left(e^{-i\left(k+\omega_{\kappa}(-k)\right) t}-1\right) g(k)\right\|^{2} d k .
$$

Отсюда следует значение среднеквадратичной нормы

$$
\left\|e^{-t \partial_{z}} \psi_{0, \kappa}^{t}-\psi_{0}\right\|^{2}=I\left(\kappa^{\circ}, \kappa\right)=\left\|e^{-t \tilde{\partial}_{z}} \tilde{\psi}_{0, \kappa}^{t}-\tilde{\psi}_{0}\right\|^{2}
$$

для функции $\psi_{0, \kappa}^{t}(z-t)$ из $\psi_{0} \in \hat{\epsilon}_{\kappa^{\circ}}^{*} \mathscr{E}_{0}^{-}$и для $\tilde{\psi}_{0, \kappa}^{t}(z+t)$ из $\tilde{\psi}_{0} \in \check{\epsilon}_{\kappa^{\circ}}^{*} \mathscr{E}_{0}^{+}$.

Для этого мы используем неравенство

$$
\left(\varkappa^{2}+\mu^{2}\right)^{1 / 2}-\varkappa<\frac{1}{2} \frac{\mu^{2}}{\varkappa} \quad \forall \varkappa>|\mu|
$$

для монотонно возрастающей функции $k+\omega_{\kappa}(-k)<\kappa^{\circ}+\omega_{\kappa}\left(-\kappa^{\circ}\right)$ от $k<\kappa^{\circ}$. Мы рассмотрим раздельно три случая релятивистской функции массы (3.1):

1) скалярный безмассовый случай $\mu_{0}=0$, где $\varepsilon(k)=|k|$;

2 ) ограниченный случай $|\mu| \leqslant m$, когда $\varepsilon(k) \leqslant \sqrt{k^{2}+m^{2}}$, как и в скалярном случае c $\mu=\mu_{0}>0$

3) общий векторньй случай $\varepsilon(k)=\left(k^{2}+\mu_{0}^{2}-\nabla^{2}\right)^{1 / 2}$.

В первом случае $k+\omega_{\kappa}(-k)=k-\kappa+|\kappa-k|=0$ для всех $\kappa \geqslant 0$ и $k<\kappa$. Таким образом, плоский волновой пропагатор

$$
\psi_{0, \kappa}^{t}(z)=\psi_{0}(z+t), \quad \tilde{\psi}_{0, \kappa}^{t}(z)=\tilde{\psi}_{0}(z-t)
$$

продолжается с помощью индуктивного предела $\kappa \rightarrow \infty$ из ортогональных классов Харди $\mathscr{E}_{0}^{\mp}$ в индуктивные пределы $\mathscr{G}_{0}^{\mp}$. В силу непрерывности они однозначно определены на всем гильбертовом пространстве $L_{\mathfrak{h}}^{2}(\mathbb{R})$, где они удовлетворяют условию $\tilde{\psi}_{0}(-z)=$ $\sigma \psi_{0}(z)$.

Во втором случае $k+\omega_{\kappa}(-k) \leqslant m^{2} /(2 \varkappa)$ для всех $\varkappa=\kappa-\kappa^{\circ}>|\mu|$ и $k<\kappa^{\circ}$.

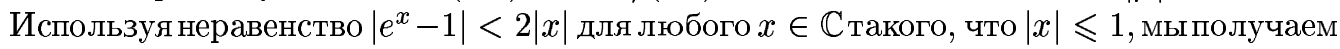
оценку

$$
\left\|I\left(\kappa^{\circ}, \kappa\right)\right\| \leqslant\left\|e^{-i\left(k+\omega_{\kappa}(-k)\right) t}-1\right\|<2|t|\left\|k+\omega_{\kappa}(-k)\right\|<|t| \frac{m^{2}}{\varkappa}
$$

для интегралов $I\left(\kappa^{\circ}, \kappa\right)$, где

$$
\|g\|^{2}=\frac{1}{2 \pi} \int\|g(k)\|^{2} d k \leqslant 1 .
$$


Следовательно, для любого $\kappa^{\circ}>0, \varepsilon>0$ и каждого $t \in \mathbb{R}$ существует $\kappa^{\prime}<\infty$ такое, что $\left\|I\left(\kappa^{\circ}, \kappa\right)\right\|<\varepsilon$ для всех $\kappa>\kappa^{\prime}$. Более точно, можно взять $\kappa^{\prime}=\kappa^{\circ}+\max \left\{m,|t| m^{2} / \varepsilon\right\}$ такое, что $\varkappa=\kappa-\kappa^{\circ}>\kappa^{\prime}-\kappa>m$ и $|t| m^{2} / \varkappa<\varepsilon$. Таким образом, распространение плоских волн действительно является ультрарелятивистским пределом решений, заданных формулами (4.9), равномерно в топологии индуктивного предела.

В третьем случае следует сделать замену $\mathfrak{h}=L^{2}\left(\mathbb{R}^{d}\right)$ в силу индуктивного предела $\mathfrak{h}^{\circ}=\bigcup \mathfrak{h}_{\kappa}$ гильбертовых пространств $\mathfrak{h}_{\kappa}$ функций в $L^{2}\left(\mathbb{R}^{d}\right)$, имеющих локализованные Фурье-образы $h(\mathbf{k})=0, \mathbf{k} \notin(-\kappa, \kappa)$ для $\kappa \in \mathbb{R}^{d}$. Тогда $\mu_{0}^{2}-\nabla^{2}<\mu_{0}^{2}+\kappa^{2}$ для каждого $\mathfrak{h}_{\kappa}$ и $\left\|I\left(\kappa^{\circ}, \kappa\right)\right\|<|t|\left(\mu_{0}^{2}+\kappa^{2}\right) / \varkappa$, если $\|g\| \leqslant 1$ для Фурье-образов $\psi_{0} \in \mathfrak{h}_{\kappa} \otimes \mathscr{E}_{\kappa^{\circ}}$ и $\tilde{\psi}_{0} \in \mathfrak{h}_{\kappa} \otimes \mathscr{E}_{\kappa^{\circ}}^{+}$, где $\mathscr{E}_{\kappa}^{\mp}-$ классы Харди в $L^{2}(\mathbb{R})$. Поэтому для любого $\kappa^{\circ}>0, \kappa \in \mathbb{R}^{d}$, $\varepsilon>0$ и каждого $t \in \mathbb{R}$ существует $\kappa^{\prime}<\infty$ такое, что $\left\|I\left(\kappa^{\circ}, \kappa\right)\right\|<\varepsilon$ для всех $\kappa>\kappa^{\prime}$, именно

$$
\kappa^{\prime}=\kappa^{\circ}+\max \left\{\sqrt{\mu_{0}^{2}+\kappa^{2}}, \frac{|t|\left(\mu_{0}^{2}+\kappa^{2}\right)}{\varepsilon}\right\} .
$$

В то же время, оценка $|t|\left(\mu_{0}^{2}+\kappa^{2}\right) /\left(\kappa-\kappa^{\circ}\right)$ теперь зависит от $\kappa$, определяющего выбор $g(k)$ в $\mathfrak{h}^{\circ}$ для произвольного $k<\kappa^{\circ}$. Это доказьвает, что пропагатор плоской волны является ультрарелятивистским индуктивньп пределом (4.9) также и в векторном случае, но не в равномерном, а в сильном смысле.

Поэтому краевая задача (4.7) в ультрарелятивистском пределе унитарно эквивалентна плоским пропагаторам (4.2) пары обратных волн $\psi_{0}, \tilde{\psi}_{0}$ с условием $\tilde{\psi}_{0}(-z)=$ $\sigma \psi_{0}(z)$ для всех $z \in \mathbb{R}$. В полупространстве $z \in \mathbb{R}^{+}$это, очевидно, может быть записано в виде

$$
\psi_{0}^{t}(z)=\chi_{0}^{t}(z), \quad z \geqslant 0, \quad \tilde{\psi}_{0}^{t}(-z)=\chi_{0}^{t}\left(z_{-}\right), \quad z \leqslant 0,
$$

где $\chi_{0}^{t}(z)=\chi_{0, t}(z+t)$ является отраженной приходящей волной (4.5). Возвращаясь $\mathrm{K}$ $\psi^{t}=\hat{\epsilon}_{\varkappa}^{*} \psi_{0}^{t}$ и $\tilde{\psi}^{t}=\check{\epsilon}_{\varkappa}^{*} \tilde{\psi}_{0}^{t}$, мы получим представление $(4.8)$ с

$$
\chi^{t}(z)=\epsilon_{\varkappa}^{*}(z) e^{t \partial_{z}} \epsilon_{\varkappa}(z) \chi_{t}(z)=\epsilon_{\varkappa}(t) \chi_{t}(z+t)
$$

в силу непрерьвности $\epsilon_{\varkappa}(t)$, где $\chi_{t}=\hat{\epsilon}_{\varkappa}^{*} \chi_{0, t}$ определено в (4.8).

ЗАмечАниЕ 3. Отраженная волна $\chi^{t}=\hat{1}_{0} \psi^{t}+\hat{1}_{0}^{\perp} \tilde{\psi}^{t}$ в представлении взаимодействия $\chi(t)=e^{i \hat{\gamma} t} \chi^{t}$ относительно группы сдвигов, порождаемой $\hat{\gamma}=i \partial_{z}$, удовлетворяет стохастическому уравнению

$$
d \chi(t, z)+i \varkappa \chi(t, z) d t=(\sigma-1) \chi(t, z) d 1_{t}(z), \quad t>0 .
$$

Действительно, динамическая группа $\epsilon_{\varkappa}(t)=e^{-i \varkappa t}$ унитарна в $\mathfrak{h}$. Однопараметрическая группа $e^{t \partial_{z}}$, очевидно, порождается самосопряженньм оператором $\gamma\left(i \partial_{z}\right)=i \partial_{z}$ в $L^{2}(\mathbb{R})$, которьй является символом генератора $\hat{\gamma}$ для группы сдвигов $e^{-i t \hat{\gamma}}$. Она является унитарной группой в $L_{\mathfrak{h}}^{2}(\mathbb{R})$ в силу инвариантности меры Лебега в $\mathbb{R}$ относительно сдвигов. Поэтому отраженная волна в представлении взаимодействия имеет вид

$$
\begin{aligned}
\chi(t, z) & =\chi^{t}(z-t)=\epsilon_{\varkappa}(t) \chi_{t}(z) \\
& =\epsilon_{\varkappa}^{*}(z-t) \epsilon_{\varkappa}(z) \chi_{t}(z)=e^{i(z-t) \varkappa} \chi_{0, t}(z),
\end{aligned}
$$

где $\chi_{0, t}=\chi_{0}+(\sigma-1)\left(1_{t}-1_{0}\right) \chi_{0}$ с $\chi_{0}=\hat{1}_{0} \psi_{0}+\hat{1}_{0}^{\perp} \tilde{\psi}_{0}$. Примем во внимание, что $d t d 1_{t}(z)=0$ в смысле гильбертова пространства, поскольку это равенство верно почти 
всюду в силу того, что оценка $d 1_{t}(z)=1 \gg d t \neq 0$ вьполнена только для одной точки $z=t$, имеющей нулевую меру. Отсюда мы получаем

$$
\begin{aligned}
d \chi(t, z) & =e^{i(z-t) \varkappa}\left[(\sigma-1) d 1_{t}(z) \chi_{0}(z)-i \varkappa \chi_{0, t}(z) d t\right] \\
& =\left[(\sigma-1) d 1_{t}(z)-i \varkappa d t\right] e^{i(z-t) \varkappa} \chi_{0, t}(z) \\
& =\left[(\sigma-1) d 1_{t}(z)-i \varkappa d t\right] \chi(t, z)
\end{aligned}
$$

Здесь мы использовали то, что $d 1_{t}(z)=d 1_{0}(z-t)=0$, если $z \neq t$ такое, что

$$
d 1_{t}(z) e^{i(z-t) \varkappa} \chi_{0, t}(z)=d 1_{t}(z) \chi_{0, t}(z)=d 1_{t}(z) \chi_{0, z}(z)
$$

в силу равенства $\left.\chi_{0, t}(z)\right|_{t=z}=\chi_{0}(z)$, так как $1_{t}(z)-1_{0}(z)=0$ для любых $z \geqslant t \geqslant 0$. Поэтому мы доказали, что $\chi(t, z)$ действительно удовлетворяет стохастическому уравнению с одним скачком (4.10) в гильбертовом пространстве $L_{\mathfrak{h}}^{2}(\mathbb{R}, \rho)$ начальных условий $\chi=\hat{1}_{0} \psi+\hat{1}_{0}^{\perp} \tilde{\psi}$ относительно унитарной группы $e^{t \partial_{z}}$.

Возвращаясь к обозначениям п. $1 \varkappa=\hbar^{-1} H, \sigma=S$ для гильбертова пространства $\mathfrak{h}=\mathscr{H}$, мы получаем уравнение (2.2) для унитарного коцикла $V(t, s)=e^{-t \partial_{z}} V^{t}$, где $V^{t}=S^{\hat{1}_{0}} e^{t\left(\partial_{z}-i \hbar^{-1} H\right)} S^{-\hat{1}_{0}}$, как квантово-механическую аппроксимацию. Именно, модель с простейшим гамильтонианом, использованная для интерпретации разрывной стохастической эволюции в терминах сильно непрерьвных унитарных разрешаюших групп для краевой задачи Дирака в дополнительной размерности, является в действительности ультрарелятивистским индуктивным пределом краевых задач для уравнения Шрёдингера с ограниченным снизу гамильтонианом $H_{\kappa}(p)=\hbar \omega_{\kappa, \varkappa}\left(-\hbar^{-1} p\right)$.

\section{СПИСОК ЦИТИРОВАННОЙ ЛИТЕРАТУРЫ}

[1] Albeverio S., Kolokoltsov V. N., Smolyanov O. G. Continuous quantum measurement: local and global approaches // Rev. Math. Phys. 1997. V. 9. № 8. P. 907-920.

[2] Belavkin V.P. A dynamical theory of quantum measurement and spontaneous localization // Russian J. Math. Phys. 1995. V. 3. № 1. P. 3-23.

[3] Accardi L., Alicki R., Frigerio A., Lu Y. G. An invitation to the weak coupling and low density limits // Quantum Probability and Related Topics. 1991. V. 6. P. 3-61.

[4] Belavkin V.P. A stochastic Hamiltonian approach for quantum jumps, spontaneous localizations, and continuous trajectories // Quantum Semiclass. Opt. 1996. V. 8. P. 167-187.

[5] Belavkin V.P. Nondemolition principle of quantum measurement theory // Foundations Phys. 1994. V. 24. № 5. P. 685-714.

[6] Чеботарев А. М. Квантовое стохастическое уравнение унитарно эквивалентно симметричной краевой задаче для уравнения Шрёдингера // Матем. заметки. 1997. Т. 61. № 4. C. $510-518$.

[7] Hudson R. L., Parthasarathy K. R. Quantum Ito's formula and stochastic evolutions // Comm. Math. Phys. 1984. V. 93. № 3. P. 301-323.

Школа математики, Ноттингемский университет, Великобритания

E-mail: vpb@maths.nott.ac.uk 